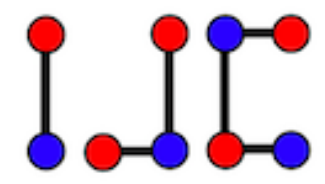

\title{
New families of star-supermagic graphs
}

\author{
Anak Agung Gede Ngurah \\ Department of Civil Engineering, Universitas Merdeka Malang \\ Jl. Taman Agung 1 Malang, Indonesia \\ aag.ngurah@unmer.ac.id
}

\begin{abstract}
A simple graph $G$ admits a $K_{1, n}$-covering if every edge in $E(G)$ belongs to a subgraph of $G$ isomorphic to $K_{1, n}$. The graph $G$ is $K_{1, n}$-supermagic if there exists a bijection $f: V(G) \cup$ $E(G) \rightarrow\{1,2,3, \cdots,|V(G) \cup E(G)|\}$ such that for every subgraph $H^{\prime}$ of $G$ isomorphic to $K_{1, n}$, $\sum_{v \in V\left(H^{\prime}\right)} f(v)+\sum_{e \in E\left(H^{\prime}\right)} f(e)$ is a constant and $f(V(G))=\{1,2,3, \cdots,|V(G)|\}$. In such a case, $f$ is called a $K_{1, n}$-supermagic labeling of $G$. In this paper, we give a method how to construct $K_{1, n}$-supermagic graphs from the old ones.
\end{abstract}

Keywords: $K_{1, n}$-covering, $K_{1, n}$-supermagic labeling, $K_{1, n}$-supermagic graph

Mathematics Subject Classification : 05C78

DOI: $10.19184 /$ ijc.2020.4.2.4

\section{Introduction}

In this paper, we consider finite and simple graphs $G$ with the vertex and edge sets $V(G)$ and $E(G)$, respectively. The number of vertices (edges) in the graph $G$ is called order (size) of $G$. Let $H$ be a given graph. An edge-covering of $G$ is a family of subgraphs $H_{1}, \ldots, H_{k}$ such that each edge in $E(G)$ belongs to at least one of the subgraphs $H_{i}, 1 \leq i \leq k$. Then it is said that $G$ admits an $\left(H_{1}, \ldots, H_{k}\right)$-(edge)covering. If every $H_{i}, 1 \leq i \leq k$, is isomorphic to the graph $H$, then $G$ admits an $H$-covering. Suppose $G$ admits an $H$-covering. A total labeling $f: V(G) \cup E(G) \rightarrow\{1,2,3, \ldots,|V(G) \cup E(G)|\}$ is called an H-magic labeling of $G$ if for every subgraph $H^{\prime}$ of $G$ isomorphic to $H, \sum_{v \in V\left(H^{\prime}\right)} f(v)+\sum_{e \in E\left(H^{\prime}\right)} f(e)=c_{f}$ is a constant. The constant $c_{f}$ is called magic constant of the labeling $f$. An $H$-magic labeling $f$ is called an

Received: 27 December 2019, Revised: 13 December 2020, Accepted: 17 December 2020. 
$H$-supermagic labeling if $f(V(G))=\{1,2,3, \ldots,|V(G)|\}$. A graph that admits $H$-(super)magic labelings is called $H$-(super)magic. In this paper, we consider such a labeling when $H$ is a star $K_{1, n}$.

The $H$-(super)magic labeling was first introduced and studied by Gutiérrez and Lladó [3] in 2005 where $H$-supermagic labelings for stars, complete bipartite graphs, paths and cycles are considered. In [7], Lladó and Moragas studied $C_{n}$-supermagic labeling of some graphs. They proved that the wheel $W_{n}$, the windmill $W(r, k)$, and the prism $C_{n} \times P_{2}$ are $C_{h}$-supermagic for some $h$. Cycles-supermagic labeling of chain graphs $k C_{n}$-path, triangle ladders $\mathrm{TL}_{n}$, grids $P_{m} \times$ $P_{n}$, for $n=2,3,4,5$, fans $F_{n}$, and books $B_{n}$ can be found in [8]. The complete results on these labelings can be found in [2].

For $H \cong P_{2}$, an $H$-supermagic graph is also called a super edge-magic graph. The notion of a super edge-magic graph was introduced by Enomoto at al [1] as a particular type of edge-magic graph given by Rosa [5]. For further information about (super) edge-magic graphs, see [2]. The $H$-magic labeling is related to a face-magic labeling of a plane graph introduced by Lih [6]. A total labeling $f$ of a plane graph is said to be face-magic if for every positive integer $s$, all $s$-sided faces have the same weight. The weight of a face under the labeling $f$ is the sum of labels carried by the edges and vertices surrounding it. Lih [6] allows different weights for different $s$. When a plane graph $G$ contains only $n$-sided faces then face-magic labeling of $\mathrm{G}$ is also $C_{n}$-magic labeling. Other results about this labeling can be found in, for instance, [2].

In this paper, we give a method how to construct star-supermagic graphs from the old ones. Based on this, we have new families of star-supermagic graphs.

\section{The Results}

In this section, we propose a method for constructing new star-supermagic graphs from certain star-supermagic graphs. To do this, we need the the following notations. The sum of all vertex and edge labels on $H$ (under a labeling $f$ ) is denoted by $\sum f(H)$. For any two integers $n<m$, the set of all consecutive integers from $n$ to $m$ is denoted by $[n, m]$. For any set $X \subset \mathbb{N}$, the set of natural numbers, we write $\Sigma X=\Sigma_{x \in X} x$. For any integer $k, X+k=\{x+k: x \in X\}$. Thus $k+[n, m]$ is the set of consecutive integers from $k+n$ to $k+m$. It is easy to check that $\Sigma(X+k)=k|X|+\Sigma X$. Furthermore, we also need the concept of a $k$-balanced set. $\mathbb{P}=\left\{X_{1}, X_{2}, \ldots, X_{k}\right\}$ is said to be an equipartition of a set of integers $X$ if $X_{1}, X_{2}, \ldots, X_{k}$ are non-empty disjoint subsets of $X$ whose union is $X$ and, for $i \in[1, k],\left|X_{i}\right|=\frac{|X|}{k}$. The set $X$ is said to be $k$-balanced if there exists an equipartition $\mathbb{P}=\left\{X_{1}, X_{2}, \ldots, X_{k}\right\}$ of $X$ with the property that $\Sigma X_{i}=\frac{\Sigma X}{k}, i \in[1, k]$.

Lemma 2.1. For any positive integers $k$ and $m$, the set $X=[1,2 k m]$ is $k$-balanced.

Proof. For every $i \in[1, k]$, define $A_{i}=[(i-1) m+1, i m]$ and $B_{i}=k m+A_{k+1-i}$. For every $i \in[1, k]$, let $C_{i}=A_{i} \cup B_{i}$. It can be checked that for $i \neq j, C_{i} \cap C_{j}=\emptyset, \bigcup_{i=1}^{k} C_{i}=X$, and for $i \in[1, k],\left|C_{i}\right|=2 m$. So, $\mathbb{P}=\left\{C_{1}, C_{2}, \ldots, C_{k}\right\}$ is an equipartition of $X$. Furthermore, for every $i \in[1, k]$, it can be checked that $\sum C_{i}=m(2 k m+1)$. Thus, $X$ is $k$-balanced.

For example, let $k=m=3$, and thus $X=[1,18]$. Then $A_{1}=\{1,2,3\}, A_{2}=\{4,5,6\}$, and $A_{3}=\{7,8,9\} . \quad B_{1}=\{16,17,18\}, B_{2}=\{13,14,15\}$, and $B_{3}=\{10,11,12\}$. The 
equipartition subsets of $X$ are $C_{1}=\{1,2,3,16,17,18\}, C_{2}=\{4,5,6,13,14,15\}$, and $C_{3}=$ $\{7,8,9,10,11,12\}$. Here, $\sum C_{1}=\sum C_{2}=\sum C_{3}=57$.

Corollary 2.1. For any positive integers $k, m$, and $p$, the set $Y=[p+1,2 k m+p]$ is $k$-balanced.

Proof. An equipartition of $Y$ is $\left\{D_{1}, D_{2}, \ldots, D_{k}\right\}$, where $D_{i}=p+C_{i}, i \in[1, k]$, and $C_{i}$ is defined as in the proof of Lemma 2.1.

Theorem 2.1. Let $G$ be a graph with of order $p$ and size $q$ edges and admits a $K_{1, \Delta(G)}$-covering, where $\Delta(G)$ is maximum degree of $G$. Let $H$ be a graph formed from $G$ by attaching $m \geq 1$ pendants to every vertex $v$ of $G$ whose degree $\operatorname{deg}(v)=\Delta(G)$. If $G$ is $K_{1, \Delta(G) \text {-supermagic, then }}$

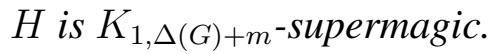

Proof. Let $G$ be a $K_{1, \Delta(G)}$-supermagic graph with a $K_{1, \Delta(G)}$-supermagic labeling $f$. Let $v_{1}, v_{2}, \ldots$, $v_{k}$ be vertices of $G$ such that $\operatorname{deg}\left(v_{i}\right)=\Delta(G), i \in[1, k]$. Then, for every $i \in[1, k]$ we have

$$
c_{f}=f\left(v_{i}\right)+\sum_{u \in N\left(v_{i}\right)} f(u)+\sum_{u \in N\left(v_{i}\right)} f\left(u v_{i}\right),
$$

where $N\left(v_{i}\right)=\left\{u: u v_{i} \in E(G)\right\}$.

Next, define $H$ as a graph with

$$
\begin{gathered}
V(H)=V(G) \cup\left\{v_{i}^{j}: i \in[1, k], j \in[1, m]\right\}, \\
E(H)=E(G) \cup\left\{v_{i} v_{i}^{j}: i \in[1, k], j \in[1, m]\right\} .
\end{gathered}
$$

Thus, $H$ is a graph of order $p+k m$ and size $q+k m$. Additionally, $H$ is a graph with maximum degree $\Delta(G)+m$. Since $G$ admits a $K_{1, \Delta(G)}$-covering and based on how $H$ is constructed, then $H$ admits a $K_{1, \Delta(G)+m \text {-covering. }}$

Let $U_{1}=[1, p], U_{2}=[p+1,2 k m+p]$, and $U_{3}=[2 k m+p+1,2 k m+p+q]$. So, $U_{1}, U_{2}, U_{3}$ is a partition of $[1,2 k m+p+q]$. By corollary 1 , the set $U_{2}=[p+1,2 k m+p]$ is $k$-balanced. For every $i \in[1, k]$, let $D_{i}$ be balanced subsets of $U_{2}$, where $D_{i}$ is defined as in the proof of Lemma 2 .

Next, define a total labeling

$$
g: V(H) \cup E(H) \rightarrow[1, p+q+2 k m]
$$

as follows.

$$
g(x)= \begin{cases}f(x), & \text { for } x \in V(G), \\ 2 k m+f(x), & \text { for } x \in E(G) .\end{cases}
$$

Under the labeling $g, g(V(G))=[1, p]$ and $g(E(G))=[2 k m+p+1,2 k m+p+q]$. Label the remaining $2 \mathrm{~km}$ pendant vertices and $2 \mathrm{~km}$ pendant edges of $H$, as follows. For $i \in[1, k]$, label $\left\{v_{i}^{j}: j \in[1, m]\right\} \cup\left\{v_{i} v_{i}^{j}: j \in[1, m]\right\}$ with the elements of $D_{i}$ such that the label of $v_{i}^{j}$ less than the label of $v_{i} v_{i}^{j}$. Thus, under the labeling $g, g(V(H))=[1, p+k m]$ and $g(E(H))=$ $[k m+p+1, p+q+2 k m]$. 
Next, we show that $g$ is a $K_{1, \Delta(G)+m}$-supermagic labeling of $H$. For every $i \in[1, k]$,

$$
\begin{aligned}
c_{g}= & g\left(v_{i}\right)+\sum_{u \in N\left(v_{i}\right)} g(u)+\sum_{u \in N\left(v_{i}\right)} g\left(u v_{i}\right) \\
= & g\left(v_{i}\right)+\sum_{u \in N\left(v_{i}\right) \cap V(G)} g(u)+\sum_{u \in N\left(v_{i}\right) \cap V(G)} g\left(u v_{i}\right) \\
& +\sum_{j=1}^{m} g\left(v_{i}^{j}\right)+\sum_{j=1}^{m} g\left(v_{i} v_{i}^{j}\right) \\
= & f\left(v_{i}\right)+\sum_{u \in N\left(v_{i}\right)} f(u)+\sum_{u \in N\left(v_{i}\right)}\left[2 k m+f\left(u v_{i}\right)\right] \\
& +\sum D_{i} \\
= & c_{f}+(2 k \Delta(G)+2 p+1) m+2 k m^{2} .
\end{aligned}
$$

Hence, $g$ is a $K_{1, \Delta(G)+m}$-supermagic labeling of $H$. So, $H$ is a $K_{1, \Delta(G)+m}$-supermagic graph.

Illustrations of Theorem 2.1 for case $\Delta(G)=2, p=k=5$, and $m=1$ is given in Figure 1, and for case $\Delta(G)=2, p=7, k=5$, and $m=2$ is given in Figure 2 .

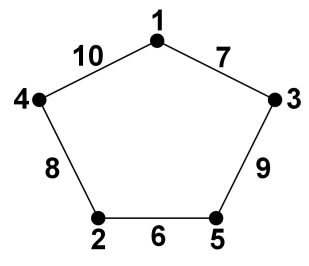

(a)

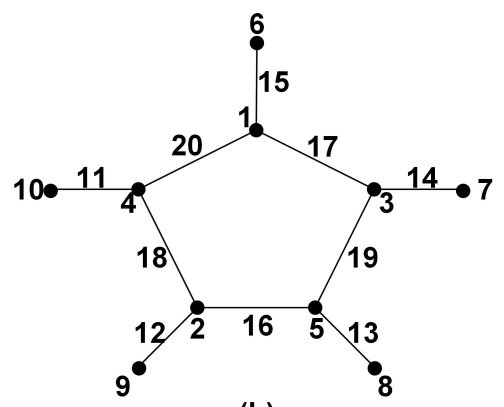

(b)

Figure 1. (a) The $K_{1,2}$-supermagic labeling of $C_{5}$ with the magic constant 25. (b) The $K_{1,3}$-supermagic labeling of the graph which is obtained by attaching a pendant to every vertex of $C_{5}$ with the magic constant 66 .

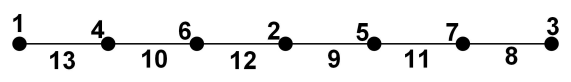

(a)

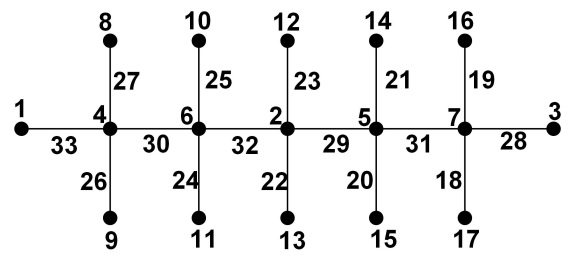

(b)

Figure 2. (a). A $K_{1,2}$-supermagic labeling of $P_{7}$ with the magic constant 34. (b) A $K_{1,4}$-supermagic labeling of a caterpillar which is formed by attaching two pendants to every vertices of $P_{7}$ except the pendants vertices with the magic constant 144.

In [3], Gutiérrez and Lladó proved the following results. The cycle $C_{n}$ is $P_{t}$-supermagic for any $t \in[2, n-1]$ such that $\operatorname{gcd}(n, t(t-1))=1$, and $P_{n}$ is $P_{h}$-supermagic for every $h \in[2, n]$. 
In particular, they proved that the cycle $C_{n}$ is $P_{3} \cong K_{1,2}$-supermagic for any $n>3$ such that $\operatorname{gcd}(n, 6)=1$, and $P_{n}$ is $P_{3}$-supermagic for every $n \geq 3$. As a consequence of these results and Theorem 2.1, we have the following corollaries.

Corollary 2.2. For any $n>3$ such that $\operatorname{gcd}(n, 6)=1$, and $m \geq 1$, the corona product of $C_{n}$ and $m K_{1}, C_{n} \odot m K_{1}$, is a $K_{1, m+2}$-supermagic graph.

Corollary 2.3. For $n \geq 3$ and $m \geq 1$, the caterpillar formed by attaching $m$ pendant edges to every vertex of degree two of the path $P_{n}$ is a $K_{1, m+2}$-supermagic graph.

The open problem related to the $K_{1, m+2}$-supermagic labeling of $C_{n} \odot m K_{1}$ is as follows.

Problem 1. For any $n>3$ such that $\operatorname{gcd}(n, 6) \neq 1$, and $m \geq 1$, determine whether there is a $K_{1, m+2}$-supermagic labeling of $C_{n} \odot m K_{1}$.

In [4], Jeyanthi and Selvagopal proved the following results.

Theorem 2.2. [4] Let $H_{1}, H_{2}, \ldots, H_{n}$ be $n$ disjoint copies of star $K_{1, n}$ and $G_{1}$ be the graph obtained by joining a new vertex to a pendant vertex of $H_{i}, i \in[1, n]$. Then $G_{1}$ is a $K_{1, n}$-supermagic graph.

Theorem 2.3. [4] Let $H_{1}, H_{2}, \ldots, H_{n+1}$ be $n+1$ disjoint copies of star $K_{1, n}$ and $G_{2}$ be the graph obtained by joining a new vertex to the center vertex of $H_{i}, i \in[1, n+1]$. Then $G_{2}$ is a $K_{1, n+1^{-}}$ supermagic graph.

Again, as a consequence of these results and Theorem 2.1, we have the following corollaries.

Corollary 2.4. For $m \geq 1$, the graphs $G_{1}^{*}$ formed by attaching $m$ pendant edges to every vertex of degree $n$ of $G_{1}$ is a $K_{1, n+m}$-supermagic graph.

Corollary 2.5. For $m \geq 1$, the graphs $G_{2}^{*}$ formed by attaching $m$ pendant edges to every vertex of degree $n+1$ of $G_{2}$ is a $K_{1, n+m+1}$-supermagic graph.

Next, we show the existence of a $K_{1, n}$-supermagic labeling of two classes of graphs for some integers $n$. Let $k \geq 1$ be an integer. Let $G_{k}$ be a graph with $V\left(G_{k}\right)=\left\{x_{i}, y_{i}: i \in[1, k+2]\right\} \cup\left\{c_{i}\right.$ : $i \in[1, k+1]\}$ amd $E\left(G_{k}\right)=\left\{x_{i} c_{i}, y_{i} c_{i}: i \in[1, k+1]\right\} \cup\left\{c_{i} x_{i+1}, c_{i} y_{i+1}: i \in[1, k+1]\right\}$. Thus, $G_{k}$ is a graph of order $3 k+5$ and size $4 k+4$, and it is obtained from a chain graph $k C_{4}$-path by attaching two pendants to the vertices $c_{1}$ and $c_{k+1}$, respectively.

Theorem 2.4. For every positive integer $k \geq 1$, the graph $G_{k}$ is $K_{1,4}$-supermagic.

Proof. Define a vertex labeling $f_{1}: V\left(G_{k}\right) \longrightarrow[1,3 k+5]$ as follows.

$$
f_{1}(u)= \begin{cases}i, & \text { if } u=x_{i}, i \text { is odd, } \\ i, & \text { if } u=y_{i}, i \text { is even, } \\ \frac{1}{2}(3 k+8-i), & \text { if } u=x_{i}, i \text { is even, } k \text { is even } \\ \frac{1}{2}(4 k+9-i), & \text { if } u=y_{i}, i \text { is odd, } k \text { is even, } \\ \frac{1}{2}(4 k+10-i), & \text { if } u=x_{i}, i \text { is even, } k \text { is odd } \\ \frac{1}{2}(3 k+8-i), & \text { if } u=y_{i}, i \text { is odd, } k \text { is odd } \\ 3 k+6-i, & \text { if } u=c_{i}, i \in[1, k+1]\end{cases}
$$


Next, for every $i \in[1, k+1]$, define an edge labeling $f_{2}: E\left(G_{k}\right) \longrightarrow[1,4 k+4]$ as follows.

$$
f_{2}(u)= \begin{cases}2 i-1, & \text { if } u=x_{i} c_{i} \\ 2 i, & \text { if } u=c_{i} x_{i+1} \\ 4 k+6-2 i, & \text { if } u=y_{i} c_{i} \\ 4 k+5-2 i, & \text { if } u=c_{i} y_{i+1}\end{cases}
$$

For every $i \in[1, k+1]$, let $K_{1,4}^{(i)}$ be the sub-stars of $G_{k}$ with vertex set $V\left(K_{1,4}^{(i)}\right)=\left\{c_{i}, x_{i}, x_{i+1}, y_{i}, y_{i+1}\right\}$ and edge set $E\left(K_{1,4}^{(i)}\right)=\left\{c_{i} x_{i}, c_{i} x_{i+1}, c_{i} y_{i}, c_{i} y_{i+1}\right\}$. It can be checked that for every $i \in[1, k+1]$,

$$
f_{1}\left(K_{1,4}^{(i)}\right)=f_{1}\left(c_{i}\right)+f_{1}\left(x_{i}\right)+f_{1}\left(y_{i}\right)+f_{1}\left(x_{i+1}\right)+f_{1}\left(y_{i+1}\right)=\left\lfloor\frac{1}{2}(13 k+31)\right\rfloor
$$

and

$$
f_{2}\left(K_{1,4}^{(i)}\right)=f_{2}\left(c_{i} x_{i}\right)+f_{2}\left(c_{i} y_{i}\right)+f_{2}\left(c_{i} x_{i+1}\right)+f_{2}\left(c_{i} y_{i+1}\right)=8 k+10
$$

Finally, define a total labeling $f_{3}: V\left(G_{k}\right) \cup E\left(G_{k}\right) \longrightarrow[1,7 k+9]$ as follows.

$$
f_{3}(u)= \begin{cases}f_{1}(u), & \text { if } u \in V\left(G_{k}\right) \\ 3 k+5+f_{2}(u), & \text { if } u \in E\left(G_{k}\right)\end{cases}
$$

It is easy to verify that, for every $i \in[1, k+1], \sum f_{3}\left(K_{1,4}^{(i)}\right)=f_{1}\left(K_{1,4}^{(i)}\right)+12 k+20+f_{2}\left(K_{1,4}^{(i)}\right)=$ $\left\lfloor\frac{1}{2}(53 k+91)\right\rfloor$.

As a direct consequence of this result and Theorem 2.1, we have the following corollary.

Corollary 2.6. For any integers $k \geq 1$ and $m \geq 1$, the graph formed by attaching $m$ pendants to every vertex of degree four of the graph $G_{k}$ is a $K_{1, m+4}$-supermagic graph.

Next, we consider of $K_{1,3}$-supermagic labelings of a ladder minus two edges. First, we define the ladder $L_{n}=P_{n} \times P_{2}, n \geq 3$, as a graph with vertex set $V\left(L_{n}\right)=\left\{x_{i}, y_{i}: i \in[1, n]\right\}$ and edge set $E\left(L_{n}\right)=\left\{x_{i} y_{i}: i \in[1, n]\right\} \cup\left\{x_{i} x_{i+1}, y_{i} y_{i+1}: i \in[1, n-1]\right\}$. For any integer $n \geq 3$, let $H_{n}=L_{n}-\left\{x_{1} y_{1}, x_{n} y_{n}\right\}$. Thus, $H_{n}$ is a graph with $V\left(H_{n}\right)=V\left(L_{n}\right)$ and $E\left(H_{n}\right)=$ $E\left(L_{n}\right)-\left\{x_{1} y_{1}, x_{n} y_{n}\right\}$. In the following theorem, we show that $H_{n}$ is $K_{1,3}$-supermagic for every $n \geq 3$.

Theorem 2.5. For any integer $n \geq 3, H_{n}$ is $K_{1,3}$-supermagic.

Proof. Define a vertex labeling $g_{1}: V\left(H_{n}\right) \longrightarrow[1,2 n]$ as follows.

Case $n \equiv 0,1 \bmod 4$.

$$
g_{1}(u)= \begin{cases}\left\lfloor\frac{1}{2}(3 n+3-i)\right\rfloor, & \text { if } u=x_{i}, i \equiv 0 \bmod 4 \\ \frac{1}{2}(i+1), & \text { if } u=x_{i}, i \equiv 1 \bmod 4 \\ \frac{1}{2}(4 n+2-i), & \text { if } u=x_{i}, i \equiv 2 \bmod 4 \\ \left\lfloor\frac{1}{2}(n+2+i)\right\rfloor, & \text { if } u=x_{i}, i \equiv 3 \bmod 4 \\ \frac{1}{2}(4 n+2-i), & \text { if } u=y_{i}, i \equiv 0 \bmod 4 \\ \left\lfloor\frac{1}{2}(n+2+i)\right\rfloor, & \text { if } u=y_{i}, i \equiv 1 \bmod 4 \\ \left\lfloor\frac{1}{2}(3 n+3-i)\right\rfloor, & \text { if } u=y_{i}, i \equiv 2 \bmod 4 \\ \frac{1}{2}(i+1), & \text { if } u=y_{i}, i \equiv 3 \bmod 4\end{cases}
$$


Case $n \equiv 2,3 \bmod 4$.

$$
g_{1}(u)= \begin{cases}\frac{1}{2}(4 n+2-i), & \text { if } u=x_{i}, i \equiv 0 \bmod 4 \\ \frac{1}{2}(i+1), & \text { if } u=x_{i}, i \equiv 1 \bmod 4 \\ \left\lfloor\frac{1}{2}(3 n+3-i)\right\rfloor, & \text { if } u=x_{i}, i \equiv 2 \bmod 4 \\ \left\lfloor\frac{1}{2}(n+2+i)\right\rfloor, & \text { if } u=x_{i}, i \equiv 3 \bmod 4 \\ \left\lfloor\frac{1}{2}(3 n+3-i)\right\rfloor, & \text { if } u=y_{i}, i \equiv 0 \bmod 4 \\ \left\lfloor\frac{1}{2}(n+2+i)\right\rfloor, & \text { if } u=y_{i}, i \equiv 1 \bmod 4 \\ \frac{1}{2}(4 n+2-i), & \text { if } u=y_{i}, i \equiv 2 \bmod 4 \\ \frac{1}{2}(i+1), & \text { if } u=y_{i}, i \equiv 3 \bmod 4\end{cases}
$$

It is easy to verify that for $i \in[2, n-1], g_{1}\left(x_{i-1}\right)+g_{1}\left(x_{i}\right)+g_{1}\left(x_{i+1}\right)+g_{1}\left(y_{i}\right)=g_{1}\left(y_{i-1}\right)+$ $g_{1}\left(y_{i}\right)+g_{1}\left(y_{i+1}\right)+g_{1}\left(x_{i}\right)$ is $4 n+3$, if $n$ is even and $4 n+4$, if $n$ is odd.

Next, define an edge labeling $g_{2}: E\left(H_{n}\right) \longrightarrow[1,3 n-4]$ as follows.

$$
g_{2}(u)= \begin{cases}\frac{1}{2}(i+1), & \text { if } u=x_{i} x_{i+1}, i \text { is odd } \\ \left\lfloor\frac{1}{2}(3 n-2+i)\right\rfloor, & \text { if } u=x_{i} x_{i+1}, i \text { is even, } \\ \frac{1}{2}(2 n-1+i), & \text { if } u=y_{i} y_{i+1}, i \text { is odd } \\ \left\lfloor\frac{1}{2}(n+i)\right\rfloor, & \text { if } u=y_{i} y_{i+1}, i \text { is even, } \\ 3 n-2-i, & \text { if } u=x_{i} y_{i}, i \in[2, n-1]\end{cases}
$$

It can be checked that for $i \in[2, n-1], g_{1}\left(x_{i-1} x_{i}\right)+g_{1}\left(x_{i} x_{i+1}\right)+g_{1}\left(x_{i} y_{i}\right)=g_{1}\left(y_{i-1} y_{i}\right)+$ $g_{1}\left(y_{i} y_{i+1}\right)+g_{1}\left(y_{i} x_{i}\right)$ is $\frac{1}{2}(9 n-6)$, if $n$ is even and $\frac{1}{2}(9 n-7)$, if $n$ is odd.

At last, define a total labeling $g_{3}: V\left(H_{n}\right) \cup E\left(H_{n}\right) \longrightarrow[1,5 n-4]$ as follows.

$$
g_{3}(u)= \begin{cases}g_{1}(u), & \text { if } u \in V\left(H_{n}\right), \\ 2 n+g_{2}(u), & \text { if } u \in E\left(H_{n}\right) .\end{cases}
$$

It is a routine procedure to check that $g_{3}$ is a $K_{1,3}$-supermagic labeling of $H_{n}$ where for every subgraph $H^{\prime}$ of $H_{n}$ isomorphic to $K_{1,3}, \sum f_{3}\left(H^{\prime}\right)$ is $\left\lfloor\frac{1}{2}(29 n+1)\right\rfloor$.

By applying Theorem 2.1 to this result, we have the following result.

Corollary 2.7. For any integers $n \geq 3$ and $m \geq 1$, the graph formed by attaching $m$ pendant edges to every vertex of degree three of the graph $H_{n}$ is a $K_{1, m+3}$-supermagic graph.

Problem 2. Investigate the existence of $K_{1, n}$-supermagic labelings of other classes of graphs.

\section{Acknowledgement}

The author would like to thank to the reviewer for his/her valuable comments and suggestions.

The author has been supported "Penelitian Dasar Unggulan Perguruan Tinggi 2019", Nomor 229/SP2H/LT/DPM/2019, 11 Maret 2019; Nomor 016/SP2H/LT/MULTI/L7/2019, 26 Maret 2019; Nomor 57/Kontrak/LPPM/UM/III/2019, 28 Maet 2019, from the Directorate General of Higher Education, Indonesia. 
New families of star-supermagic graphs | A.A.G. Ngurah

\section{References}

[1] H. Enomoto, A. Llado, T. Nakamigawa, and G. Ringel, Super edge magic graphs, SUT J. Math. 34 (1998), 105-109.

[2] J.A. Gallian, A dynamic survey of graph labelings, Electron. J. Combin. 14 (2019) \# DS6.

[3] A. Gutiérrez and A. Lladó, Magic coverings, J. Combin. Math. Combin. Comput. 55 (2005), 43-56.

[4] P. Jeyanthi and P. Selvagopal, Construction of supermagic graphs, Communicated.

[5] A. Kotzig and A. Rosa, Magic valuation of finite graphs, Canad. Math. Bull. Vol. 13 (4), (1970), 451-461.

[6] K.W. Lih, On magic and consecutive labelings of plane graphs, Utilitas Math. 24 (1983), 165-197.

[7] A. Lladó and J. Moragas, Cycle-magic graphs, Discrete math. 307 (23), (2007), 2925-2933.

[8] A.A.G. Ngurah, A.N.M. Salman, and L. Susilowati, $H$-supermagic labelings of graphs, Discrete Math. 310 (8), 1293-1300. 